PROCEEDINGS OF THE

AMERICAN MATHEMATICAL SOCIETY

Volume 132, Number 8, Pages 2257-2263

S 0002-9939(04)07141-2

Article electronically published on March 25, 2004

\title{
THE RUSSO-DYE THEOREM IN A WEAKLY CLOSED $\mathcal{T}(\mathcal{N})$-MODULE
}

\author{
ZHE DONG
}

(Communicated by David R. Larson)

\begin{abstract}
Suppose that $\mathcal{N}$ is admissible. It is shown that the convex hull of unitary elements of a weakly closed $\mathcal{T}(\mathcal{N})$-module $\mathcal{U}$ contains the whole unit ball of $\mathcal{U}$ if and only if $\widetilde{I}=I$ and for any $N>0, \widetilde{N}>0$.
\end{abstract}

\section{INTRODUCTION}

One of the well-known results in the theory of $C^{*}$-algebras is the Russo-Dye Theorem [8: the closed convex hull of the unitary group of any $C^{*}$-algebra is the closed unit ball. A number of refinements on this theorem led to the KadisonPederson's result [5] that every strict contraction of a $C^{*}$-algebra is the mean of a finite number of unitary elements of the $C^{*}$-algebra.

In non-selfadjoint algebras, the first surprising fact resulting from the Russo-Dye Theorem was proved by Anoussis and Katsoulis 1], that is: for any nest with no finite atoms, every strict contraction in the nest algebra is the average of unitary elements in the algebra. In 2], Davidson established that the Russo-Dye Theorem holds in a nest algebra if and only if $\mathcal{N}$ is admissible. The Russo-Dye Theorem has proved very useful. It provides one means of reducing the study of a non-normal element to that of normal (unitary) elements - and the device is reasonably sensitive to norm estimates. The main purpose of this paper is to establish the Russo-Dye Theorem in a weakly closed $\mathcal{T}(\mathcal{N})$-module. To our knowledge, this is the first result of this type for a non-algebraic operator system. Though the exposition and structure of this paper follows closely those in [2], the main result of this paper is unexpected.

Let us introduce some notation and terminology. $\mathcal{H}$ represents a complex separable infinite-dimensional Hilbert space, and $\mathcal{B}(\mathcal{H})$ the algebra of bounded operators on $\mathcal{H}$. A nest $\mathcal{N}$ is a chain of closed subspaces of Hilbert space $\mathcal{H}$ containing (0) and $\mathcal{H}$ which is closed under intersection and closed span. If $N$ is an element of a nest $\mathcal{N}$, then $N_{-}$denotes the immediate predecessor of $N$. Similarly we define $N_{+}$ as the immediate successor of $N$. Say that a nest is admissible if $0_{+}$and $I_{-}^{\perp}$ are either zero or of infinite rank. If $\mathcal{N}$ is a nest, then the nest algebra $\mathcal{T}(\mathcal{N})$ is the set of all operators $T$ such that $T N \subseteq N$ for every element $N$ in $\mathcal{N}$.

Received by the editors October 19, 2000.

2000 Mathematics Subject Classification. Primary 47L75.

Key words and phrases. Russo-Dye Theorem, weakly closed $\mathcal{T}(\mathcal{N})$-module, unitary operator. 
Suppose that $N \rightarrow \widetilde{N}$ is an order homomorphism of $\mathcal{N}$ into itself (that is, $N \leq N^{\prime}$ implies $\widetilde{N} \leq \widetilde{N^{\prime}}$ ). Then the set

$$
\mathcal{U}=\{X \in \mathcal{B}(\mathcal{H}): X N \subseteq \widetilde{N}, \forall N \in \mathcal{N}\}
$$

is clearly a weakly closed (two sided) $\mathcal{T}(\mathcal{N})$-module. In [4], Erdos and Power show that every weakly closed $\mathcal{T}(\mathcal{N})$-module is of the above form, where the order homomorphism $N \rightarrow \widetilde{N}$ is left continuous and $\widetilde{0}=0$. For each $N \in \mathcal{N}$, define

$$
N_{*}=\bigwedge\left\{\widetilde{N^{\prime}}: N^{\prime}>N\right\}
$$

and

$$
N_{\sim}=\bigvee\left\{N^{\prime}: \widetilde{N^{\prime}}<N\right\}
$$

Since $\mathcal{N}$ is complete, $N_{*}$ and $N_{\sim}$ are in $\mathcal{N}$.

An interval of $\mathcal{N}$ is a projection $E=N_{2}-N_{1}$, where $N_{1}<N_{2}$ belong to $\mathcal{N}$. The support $\operatorname{supp}(e)$ in $\mathcal{N}$ of a vector $e$ is the smallest interval $E$ such that $E e=e$. Similarly the support of a projection in the diagonal algebra $\mathcal{N}^{\prime}$ is the smallest interval supporting every vector of its range. If $E, F$ are two intervals and $\mathcal{U}$ is a weakly closed $\mathcal{T}(\mathcal{N})$-module, then we say that $E \preceq F$ according to $\mathcal{U}$ if $E \mathcal{U} F=E \mathcal{B}(\mathcal{H}) F$. Note that $N_{*} \preceq N^{\perp}, N \preceq N_{\sim}^{\perp}$ and $\widetilde{N} \preceq N_{-}^{\perp}$ according to $\mathcal{U}$.

The notation $x \otimes y$ denotes the rank one operator $(x \otimes y)(z)=(z, y) x$ for $z \in \mathcal{H}$.

Throughout this paper, let $\mathcal{T}(\mathcal{N})$ be the nest algebra associated with a nest $\mathcal{N}$. Let $\mathcal{U}$ be the weakly closed $\mathcal{T}(\mathcal{N})$-module determined by a left continuous order homomorphism $N \rightarrow \widetilde{N}$ with $\widetilde{0}=0$.

\section{The Russo-Dye Theorem}

The main theorem can now be stated.

Theorem 1. Suppose that $\mathcal{N}$ is admissible. Then the following statements are equivalent:

1) every element $A \in \mathcal{U}$ such that $\|A\| \leq 1-\frac{1}{n}$ is the mean of $16 n^{2}$ unitary elements of $\mathcal{U}$;

2) the convex hull of these unitary elements in $\mathcal{U}$ contains the whole open unit ball of $\mathcal{U}$, thus the Russo-Dye Theorem holds in $\mathcal{U}$;

3) $\widetilde{I}=I$ and for any $N>0, \widetilde{N}>0$.

Proof. We first prove 1) $\Rightarrow 2$ ) and 2) $\Rightarrow 3$ ).

1) $\Rightarrow 2$ ). Obvious.

$2) \Rightarrow 3$ ). Suppose that 3 ) is not true. Then $\widetilde{I}<I$ or there exists an element $N>0$ in $\mathcal{N}$ and $\widetilde{N}=0$. We deal with the two cases separately.

i) If $\widetilde{I}<I$, let $U$ be a unitary element of $\mathcal{U}$. Thus $U I \subseteq \widetilde{I}$ and $U^{*} \widetilde{I}^{\perp} \subseteq I^{\perp}=0$, which contradicts the fact that $U^{*}$ is a unitary operator. This contradiction shows that there is no unitary operator in $\mathcal{U}$ if $\widetilde{I}<I$.

ii) If there is an element $N>0$ and $\widetilde{N}=0$, a similar argument shows that there exists no unitary operator in $\mathcal{U}$.

If 3 ) is not true, it follows from i) and ii) that there is no unitary operator in $\mathcal{U}$. This shows 2) does not hold.

The difficulties of the proof lie in 3$) \Rightarrow 1$ ). To prove this, we need to establish some auxiliary results first. 
Lemma 2. Suppose that $\mathcal{U}$ satisfies condition 3) in Theorem 1 and $Q$ is a diagonal projection of $\mathcal{T}(\mathcal{N})$. Let $\left\{f_{k}\right\}$ be an orthonormal set such that infinitely many $f_{k}$ 's belong to $N$ for any every $N>0$ in $\mathcal{N}$. Then there is a partial isometry $S$ in $\mathcal{U}$ such that $S^{*} S=Q$ and its range is spanned by a subset of the $f_{k}$ 's.

Proof. If $E=0_{*}=\bigwedge\{\tilde{N}: N>0\} \neq 0$, then $\mathcal{F}=\left\{f_{k}: f_{k}=E f_{k}\right\}$ is infinite and $E$ is of infinite rank. In this case, we construct a partial isometry $S$ in $\mathcal{B}(\mathcal{H})$ with $S^{*} S=Q$ and range spanned by a subset of $\mathcal{F}$. For any $N \in \mathcal{N} \backslash(0)$, it follows from the definition of $0_{*}$ that $\tilde{N} \geq 0_{*}=E$. Hence $S N \subseteq E \subseteq \widetilde{N}$, so $S \in \mathcal{U}$. Now we suppose that $0_{*}=0$. If $0_{+} \neq 0,0_{*}=\widetilde{0_{+}}>0$ by condition 3 ) in Theorem 1. This shows that $0_{+}=0$. Thus there is a sequence $\left\{N_{k}\right\}$ in $\mathcal{N}$ strictly decreasing with limit 0 . Choose an orthonormal basis for each $\left(N_{k}-N_{k+1}\right) Q \mathcal{H}$ starting with $N_{0}=I$, and combine them to form an orthonormal basis $\left\{e_{k}: k \geq 1\right\}$ for $Q \mathcal{H}$ with the property that $e_{k}=M_{k}^{\perp} e_{k}$ for some $M_{k} \in \mathcal{N}$ with $M_{k}>0$. Since $M_{k}>0, \widetilde{M_{k}}>0$. Hence we can choose an increasing sequence $\left\{n_{k}\right\}$ such that $f_{n_{k}}$ is contained in $\widetilde{M_{k}}$ for $k \geq 1$. The operator $S=\sum_{k \geq 1} f_{n_{k}} \otimes e_{k} \in \mathcal{U}$ is the desired partial isometry.

Lemma 3. Suppose that $\mathcal{U}$ satisfies condition 3) in Theorem 1 and $P$ is a diagonal projection in $\mathcal{T}(\mathcal{N})$. Let $\left\{e_{k}\right\}$ be an orthonormal set such that infinitely many $e_{k}$ 's belong to $N^{\perp}$ for every $N<I$ in $\mathcal{N}$. Then there is a partial isometry $S$ in $\mathcal{U}$ such that $S S^{*}=P$ and its domain is spanned by a subset of the $e_{k}$ 's.

Proof. If $I \neq I_{\sim}=\bigvee\{N: \widetilde{N}<I\}$, then $\mathcal{E}=\left\{e_{k}: I_{\sim}^{\perp} e_{k}=e_{k}\right\}$ is of infinite rank. Hence we can construct a partial isometry $S$ in $\mathcal{B}(\mathcal{H})$ with $S S^{*}=P$ and its domain spanned by a subset of $\mathcal{E}$. For any $N \in \mathcal{N}$, if $\widetilde{N}=I$, then $S^{*} \widetilde{N}^{\perp}=$ $(0) \subseteq N^{\perp}$; if $\widetilde{N}<I$, then $N \leq I_{\sim}$ and $I_{\sim}^{\perp} \leq N^{\perp}$. Since $\operatorname{Ran}\left(S^{*}\right)=\operatorname{ker}(S)^{\perp} \subseteq I_{\sim}^{\perp}$, $S^{*} \widetilde{N}^{\perp} \subseteq I_{\sim}^{\perp} \subseteq N^{\perp}$. Hence for any $N \in \mathcal{N}, S^{*} \widetilde{N}^{\perp} \subseteq N^{\perp}$ and $S N \subseteq \widetilde{N}$, so $S \in \mathcal{U}$. Now suppose that $I=I_{\sim}$. We first prove that $N<I$ is equivalent to $\widetilde{N}<I$. Suppose that $N<I$ and $\widetilde{N}=I$; thus for any $M \geq N, \widetilde{M} \geq \widetilde{N}=I$. So $I=I_{\sim}=\bigvee\left\{N^{\prime}: \widetilde{N^{\prime}}<I\right\} \leq N<I$; this contradiction shows $\widetilde{N}<I$. If $\widetilde{N}<I$ and $N=I$, we have $\widetilde{N}=\widetilde{I}=I$ by condition 3 ) in Theorem 1. This is a contradiction, so $N<I$. Hence $I=I_{\sim}=\bigvee\{N: \widetilde{N}<I\}=\bigvee\{N: N<I\}=I_{-}$. Thus there is a sequence $N_{k}$ in $\mathcal{N} \backslash I$ strictly increasing with $I$. Since the order homomorphism $N \rightarrow \widetilde{N}$ is left order continuous, we have that $\left\{\widetilde{N_{k}}\right\}$ is also increasing with limit $\widetilde{I}=I$. Since $N_{k}<I, \widetilde{N_{k}}<I$. Hence by taking a subsequence, we can suppose that $\left\{\widetilde{N}_{k}\right\}$ is strictly increasing with limit $I$. Choose an orthonormal basis for each $\left(\widetilde{N_{k+1}}-\widetilde{N_{k}}\right) P \mathcal{H}$ starting with $N_{0}=0=\widetilde{0}$, and combine them to form an orthonormal basis $\left\{f_{k}: k \geq 1\right\}$ for $P \mathcal{H}$ with the property that each $f_{k}=\widetilde{M_{k}} f_{k}$ for some $M_{k} \in \mathcal{N}$ with $M_{k}<I$. Then choose an increasing sequence $\left\{n_{k}\right\}$ such that $e_{n_{k}}$ is contained in $M_{k}^{\perp}$ for each $k \geq 1$. Then the operator $S=\sum_{k \geq 1} f_{k} \otimes e_{n_{k}} \in \mathcal{U}$ does the job.

Lemma 4. Let $\left\{e_{k}\right\}$ be an orthonormal set such that each $e_{k}=M_{k}^{\perp} e_{k}$ for some $M_{k} \in \mathcal{N}$ with $M_{k *}>0$ and infinitely many $e_{k}$ 's belong to $N^{\perp}$ for every $N<I$ in $\mathcal{N}$. Also let $\left\{f_{k}\right\}$ be an orthonormal set such that each $f_{k}=N_{k} f_{k}$ for some $N_{k} \in \mathcal{N}$ with $N_{k \sim}<I$ and infinitely many $f_{k}$ 's belong to $N$ for some $N>0$ in $\mathcal{N}$. Then 
there is a partial isometry in $\mathcal{U}$ with initial space $\operatorname{span}\left\{e_{k}: k \geq 1\right\}$ and range space $\operatorname{span}\left\{f_{k}: k \geq 1\right\}$.

Proof. This proof is a slight modification of the proof of the last situation in [2], Lemma 1.

The partial isometry $S$ is contructed using a well-known combinatoral device to determine an infinite permutation $\pi$ of $\mathbf{N}$ such that

$$
\operatorname{supp}\left(f_{\pi(k)}\right) \preceq \operatorname{supp}\left(e_{k}\right) \text { according to } \mathcal{U}, \quad \text { for all } k \in \mathbf{N} .
$$

Indeed, at the $k$ th stage, if $\pi(k)$ is not defined, choose it to be an integer $n=\pi(k)$ not yet in the range of $\pi$ such that (1) holds. This is possible since $e_{k}=M_{k}^{\perp} e_{k}$ and infinitely many $f_{n}$ 's are supported on $M_{k *}>0$. Then if $k$ is not in the range of $\pi$, choose $n=\pi^{-1}(k)$ in the same manner to be an integer on which $\pi$ is as yet undefined and for which $\operatorname{supp}\left(f_{k}\right) \preceq \operatorname{supp}\left(e_{n}\right)$ according to $\mathcal{U}$ (since $N_{k} f_{k}=f_{k}$ and infinitely many $e_{n}$ 's are supported on $N_{k \sim}^{\perp}$ ). In this way, a bijection satisfying (1) is obtained. Then the operator $S=\sum_{k \geq 1} f_{\pi(k)} \otimes e_{k} \in \mathcal{U}$ is the desired partial isometry.

Now suppose that $\mathcal{N}$ is admissible. In this case, it is easy to construct a projection $P=\sum_{-\infty}^{+\infty} E_{n}$ in $\mathcal{N}^{\prime}$ such that each $E_{n}$ is an orthonormal projection in $\mathcal{N}^{\prime}$, $E_{n} \preceq E_{n+1}$ according to $\mathcal{T}(\mathcal{N}), E_{n} \perp E_{n+1}$ and the smallest projection interval of $\mathcal{N}$ containing $P$ is the identity $I$. Such a projection will also be called admissible. Note that $\mathcal{N}$ is admissible if and only if there are admissible projections in $\mathcal{T}(\mathcal{N})$. Any admissible projection $P$ may be decomposed as a sum of countably many pairwise orthogonal admissible projections by splitting $Z$ into countably many disjoint subsets which are not bounded above or below.

Lemma 5. Suppose that $\mathcal{U}$ satisfies condition 3) in Theorem 1 and $P=\sum_{-\infty}^{+\infty} E_{n}$ is an admissible projection in $\mathcal{T}(\mathcal{N})$. Choose orthonormal bases for each of these subspaces and combine them to form an orthonormal basis $\left\{h_{k}\right\}$ for PH. Then

1) each $h_{k}=M_{k}^{\perp} h_{k}$ for some $M_{k} \in \mathcal{N}$ with $M_{k *}>0$;

2) each $h_{k}=N_{k} h_{k}$ for some $N_{k} \in \mathcal{N}$ with $N_{k \sim}<I$.

Proof. 1) We deal separately with two cases.

i) $0_{*} \neq 0$. For all $k \in \mathbf{N}, M_{k}=0$ does the job.

ii) $0_{*}=0$. It follows from a similar argument in Lemma 2 that $0_{*}=0=0_{+}$. For any $N>0, N_{*}=\bigwedge\{\widetilde{M}: M>N\} \geq \widetilde{N}>0$. Thus for each $h_{k}$, since $0_{+}=0$ and $h_{k}$ belong to some $E_{n}$, there exists some $M_{k}>0$ in $\mathcal{N}$ such that $M_{k}^{\perp} h_{k}=h_{k}$ and $M_{k *}>0$.

2) If $I_{\sim} \neq I$, for each $h_{k}$, we can take $N_{k}=I$. If $I_{\sim}=I$, by the proof of Lemma $3, I=I_{-}$. We first prove the following assertion: for any $N<I, N_{\sim}<I$.

Indeed, if there exists an element $N<I$ and $N_{\sim}=\bigvee\{M: \widetilde{M}<N\}=I=I_{-}$, then there is a sequence $\left\{M_{k}^{\prime}\right\}$ with $\widetilde{M_{k}^{\prime}}<N$ and strictly increasing with limit $I$. It follows from the left continuity of the order homomorphism that the sequence $\left\{\widetilde{M_{k}^{\prime}}\right\}$ is also increasing with limit $\widetilde{I}=I$. But this contradicts the fact $\widetilde{M_{k}^{\prime}}<N<I$.

For each $h_{k}$, since $I=I_{-}$and $h_{k}$ belongs to some $E_{n}$, there exists an element $N_{k}<I$ in $\mathcal{N}$ such that $N_{k} h_{k}=h_{k}$ and $N_{k \sim}<I$. 
Proposition 6. Suppose that $\mathcal{U}$ satisfies condition 3) in Theorem 1 and $\mathcal{N}$ is an adimissible nest with admissible projections $P$ and $Q$. Let $A$ be an element in $\mathcal{U}$ such that $A=Q^{\perp} A P^{\perp}$ and $\|A\|<1$. Then there is a unitary $U$ in $\mathcal{U}$ such that $Q^{\perp} U P^{\perp}=A$. Thus $A$ is the average of four unitaries in $\mathcal{U}$.

Proof. Let $E_{n}$ be the subintervals of $P$ ordered by $Z$ in the partial order on subintervals of $\mathcal{N}$, and choose a unit vector $e_{n}$ in the range of $E_{n}$ for $n \in Z$. Similarly, let $F_{n}$ be the subintervals of $Q$ and let $f_{n}$ be unit vectors in $F_{n} \mathcal{H}$ for $n \in Z$. Let $P_{0}$ and $P_{1}$ be the projections onto $\operatorname{span}\left\{e_{2 n}: n \in Z\right\}$ and $\operatorname{span}\left\{e_{2 n+1}: n \in Z\right\}$ respectively. Similarly define $Q_{0}$ and $Q_{1}$.

Since $A$ is a strict contraction, $P^{\perp}-A^{*} A$ and $Q^{\perp}-A A^{*}$ are positive and invertible in $\mathcal{B}\left(P^{\perp} \mathcal{H}\right)$ and $\mathcal{B}\left(Q^{\perp} \mathcal{H}\right)$ respectively. Thus by [6] there exist invertible operators $X$ in $\mathcal{T}\left(P^{\perp} \mathcal{N}\right)=P^{\perp} \mathcal{T}(\mathcal{N}) P^{\perp}$ and $Y$ in $\mathcal{T}\left(Q^{\perp} \mathcal{N}\right)=Q^{\perp} \mathcal{T}(\mathcal{N}) Q^{\perp}$ such that

$$
P^{\perp}-A^{*} A=X^{*} X \text { and } Q^{\perp}-A A^{*}=Y Y^{*}
$$

( $X^{-1}, Y^{-1}$ need not belong to the nest algebras). Using the polar decomposition for $X$ and $Y^{*}$, we obtain

$$
X=U_{1}\left(P^{\perp}-A^{*} A\right)^{\frac{1}{2}} \quad \text { and } \quad Y=\left(Q^{\perp}-A A^{*}\right)^{\frac{1}{2}} U_{2},
$$

where $U_{1}=P^{\perp} U_{1} P^{\perp}$ and $U_{2}=Q^{\perp} U_{2} Q^{\perp}$ are partial isometries in $\mathcal{B}(\mathcal{H})$. Set $T=-U_{1} A^{*} U_{2}=P^{\perp} T Q^{\perp}$.

Fix a proper element $M$ in $\mathcal{N}$ and use Lemma 2 to construct a partial isometry $W_{1}$ in $\mathcal{U}$ such that $W_{1}^{*} W_{1}=P^{\perp}$ with range contained in $\widetilde{M} Q_{0} \mathcal{H}$ and spanned by a subset of the $f_{n}$ 's. Similarly construct another partial isometry $W_{2}$ in $\mathcal{U}$ such that $W_{2} W_{2}^{*}=Q^{\perp}$, with domain contained in $M^{\perp} P_{0} \mathcal{H}$ spanned by a subset of the $e_{n}$ 's. Since $W_{1} T W_{2}=P(\widetilde{M}) W_{1} T W_{2} P(M)^{\perp}, W_{1} T W_{2} \in \mathcal{U}$. Decomposing $\mathcal{B}(\mathcal{H})$ as operators from $\mathcal{H}=P^{\perp} \mathcal{H} \oplus P \mathcal{H}$ into $\mathcal{H}=Q^{\perp} \mathcal{H} \oplus Q \mathcal{H}$, we set

$$
S=A+Y W_{2}+W_{1} X+W_{1} T W_{2} \simeq\left(\begin{array}{cc}
A & Y W_{2} \\
W_{1} X & W_{1} T W_{2}
\end{array}\right) .
$$

Since $X, Y \in \mathcal{T}(\mathcal{N})$ and $W_{1}, W_{2} \in \mathcal{U}, Y W_{2}$ and $W_{1} X$ belong to $\mathcal{U}$. Hence $S \in \mathcal{U}$. Now we compute $S^{*} S$ and $S S^{*}$ :

$$
S^{*} S \simeq\left(\begin{array}{cc}
A^{*} A+X^{*} W_{1}^{*} W_{1} X & A^{*} Y W_{2}+X^{*} W_{1}^{*} W_{1} T W_{2} \\
W_{2}^{*} Y^{*} A+W_{2}^{*} T^{*} W_{1}^{*} W_{1} X & W_{2}^{*} Y^{*} Y W_{2}+W_{2}^{*} T^{*} W_{1}^{*} W_{1} T W_{2}
\end{array}\right),
$$

with

$$
\begin{aligned}
A^{*} A+ & X^{*} W_{1}^{*} W_{1} X=A^{*} A+X^{*} X=P^{\perp} \\
A^{*} Y W_{2}+X^{*} W_{1}^{*} W_{1} T W_{2} & =A^{*} Y W_{2}+X^{*} T W_{2} \\
& =A^{*}\left(Q^{\perp}-A A^{*}\right)^{\frac{1}{2}} U_{2} W_{2}-\left(P^{\perp}-A^{*} A\right)^{\frac{1}{2}} U_{1}^{*} U_{1} A^{*} U_{2} W_{2} \\
= & {\left[A^{*}\left(Q^{\perp}-A A^{*}\right)^{\frac{1}{2}}-\left(P^{\perp}-A^{*} A\right)^{\frac{1}{2}} A^{*}\right] U_{2} W_{2}=0 ; } \\
W_{2}^{*} Y^{*} Y W_{2}+W_{2}^{*} T^{*} W_{1}^{*} W_{1} T W_{2} & =W_{2}^{*}\left(Y^{*} Y+T^{*} T\right) W_{2} \\
& =W_{2}^{*}\left[U_{2}^{*}\left(Q^{\perp}-A A^{*}\right) U_{2}+U_{2}^{*} A U_{1}^{*} U_{1} A^{*} U_{2}\right] W_{2} \\
& =W_{2}^{*}\left[U_{2}^{*}\left(Q^{\perp}-A A^{*}\right) U_{2}+U_{2}^{*} A A^{*} U_{2}\right] W_{2} \\
& =W_{2}^{*}\left(U_{2}^{*} U_{2}\right) W_{2}=W_{2}^{*} Q^{\perp} W_{2}=W_{2}^{*} W_{2} .
\end{aligned}
$$


Hence $S^{*} S=P^{\perp}+W_{2}^{*} W_{2}$ and, by a similar argument, $S S^{*}=Q^{\perp}+W_{1} W_{1}^{*}$. So $S$ is a partial isometry of $\mathcal{U}$. The projection $I-S^{*} S=P-W_{2}^{*} W_{2}$ dominates $P_{1}$ and is spanned by the properly supported projections $E_{n}$ or $E_{n}-e_{n} \otimes e_{n}$ for $n \in Z$. Similarly, $I-S S^{*}=Q-W_{1} W_{1}^{*}$ dominates $Q_{1}$ and is spanned by the properly supported projections $F_{n}$ or $F_{n}-f_{n} \otimes f_{n}$ for $n \in Z$. Thus $I-S^{*} S$ and $I-S S^{*}$ have infinite rank intersection with $N$ and $N^{\perp}$ for every $N \in \mathcal{N} \backslash(0, I)$, and their ranges are spanned by subspaces which have proper support. We choose orthonormal bases for each of these subspaces and combine them to form orthonormal bases $\left\{g_{k}\right\}$ for $I-S^{*} S$ and $\left\{h_{k}\right\}$ for $I-S S^{*}$. It follows from the proof of Lemma 5 that $\left\{g_{k}\right\}$ and $\left\{h_{k}\right\}$ satisfy the hypothesis of Lemma 4 . Hence there is a partial isometry $S_{1}$ in $\mathcal{U}$ such that $S_{1}^{*} S_{1}=I-S^{*} S$ and $S_{1} S_{1}^{*}=I-S S^{*}$. It is easy to prove that $U=S+S_{1}$ is a unitary operator of $\mathcal{U}$ and $Q^{\perp} U P^{\perp}=Q^{\perp} S P^{\perp}=A$.

Finally, define unitary operators

$$
U_{k}=\left(Q^{\perp}+i^{k} Q\right) U\left(P^{\perp}+i^{k} P\right) \in \mathcal{U}, \quad 1 \leq k \leq 4 .
$$

By computing, we have

$$
\frac{1}{4} \sum_{k=1}^{4} U_{k}=Q^{\perp} U P^{\perp}=A .
$$

Now we are in the position to complete the proof of Theorem 1.

$3) \Rightarrow 1$ ) of Theorem 1: Let $P$ and $Q$ be admissible projections. Split $P$ and $Q$ into $2 n$ orthonormal admissible projections $P_{1}, \cdots, P_{2 n}$ and $Q_{1}, \cdots, Q_{2 n}$, respectively. It follows that $X_{i}=Q_{i}^{\perp}-\frac{1}{2 n} Q^{\perp}$ and $Y_{i}=P_{i}^{\perp}-\frac{1}{2 n} P^{\perp}$ are contractions in $\mathcal{N}^{\prime}$ such that

$$
\sum_{i=1}^{2 n} X_{i}=(2 n-1) I=\sum_{i=1}^{2 n} Y_{i} .
$$

Moreover, $\left(1-\frac{1}{2 n}\right)^{2}>1-\frac{1}{n}$. Thus the operators

$$
A_{i j}=\left(\frac{2 n}{2 n-1}\right)^{2} X_{i} A Y_{j}=Q_{i}^{\perp} A_{i j} P_{j}^{\perp} \text { for } 1 \leq i, j \leq n
$$

are strict contractions in $\mathcal{U}$. By Proposition 6, each $A_{i j}$ is the mean of four unitaries $U_{i j k} \in \mathcal{U}$ for $1 \leq k \leq 4$. Hence

$$
\begin{aligned}
\frac{1}{16 n^{2}} \sum_{i=1}^{2 n} \sum_{j=1}^{2 n} \sum_{k=1}^{4} U_{i j k} & =\frac{1}{4 n^{2}} \sum_{i=1}^{2 n} \sum_{j=1}^{2 n} A_{i j} \\
& =\frac{1}{4 n^{2}}\left(\sum_{i=1}^{2 n} X_{i}\right)\left(\frac{2 n}{2 n-1}\right)\left(\sum_{j=1}^{2 n} Y_{j}\right) \\
& =\frac{1}{4 n^{2}}(2 n-1)^{2}\left(\frac{2 n}{2 n-1}\right)^{2} A=A .
\end{aligned}
$$

Now as a corollary of Theorem 1, we give a sufficient condition for the KreinMilman theorem to hold in $\mathcal{U}$.

Corollary 7. If $\mathcal{N}$ is admissible and $\mathcal{U}$ satisfies condition 3$)$ in Theorem 1 , then $\operatorname{ext}\left(\mathcal{U}_{1}\right) \neq \emptyset$ and $\overline{\operatorname{co}} \operatorname{ext}\left(\mathcal{U}_{1}\right)=\mathcal{U}_{1}$. 
Proof. It follows from Theorem 1 that the closed convex hull of the unitary elements in $\mathcal{U}$ equals the closed unit ball of $\mathcal{U}$, and Theorem 3.3 in [3] shows that each unitary element in $\mathcal{U}$ is an extreme point of $\mathcal{U}_{1}$. Then $\operatorname{ext}(\mathcal{U}) \neq \emptyset$ and $\overline{\operatorname{co}} \operatorname{ext}\left(\mathcal{U}_{1}\right)=\mathcal{U}_{1}$.

Remark 8. Consider the case when $\mathcal{N}$ is not admissible.

If $\mathcal{N}$ is not admissible, it follows from [2] that the Russo-Dye Theorem does not hold in $\mathcal{T}(\mathcal{N})$. Furthermore, we can easily prove:

Suppose that $\mathcal{N}$ is not admissible. If $\widetilde{0_{+}} \leq 0_{+}$or $\widetilde{I_{-}} \leq I_{-}$, then the Russo-Dye Theorem does not hold in $\mathcal{U}$.

In light of Theorem 1 , it is natural to ask if $\widetilde{0_{+}}>0_{+}$and $\widetilde{I_{-}}=I$ is a sufficient condition for the Russo-Dye Theorem to hold in $\mathcal{U}$. This seems to be a challenging question. We shall continue the investigation.

\section{REFERENCES}

1. M.Anoussis and E.Katsoulis, A nonself-adjoint Russo-Dye Theorem, Math. Ann. 304 (1996), 685-699. MR 97f:47042

2. K.R.Davidson, The Russo-Dye theorem in nest algebras, Proc. Amer. Math. Soc. 126 (1998), 3055-3059. MR 2000f:47109

3. Dong Zhe and Lu Shijie, Extreme points of weakly closed $\mathcal{T}(\mathcal{N})$-modules, Proc. Amer. Math. Soc. 130 (2002), 461-469. MR 2002m:47086

4. J.A.Erdos and S.C.Power, Weakly closed ideals of nest algebras, J. Operator Theory 7 (1982), 219-235. MR 84a:47056

5. R.V.Kadison and G.K.Pedersen, Means and convex combinations of unitary operators, Math. Scand. 57 (1985), 245-266. MR 87g:47078

6. D.R.Larson, Nest algebras and similarity transforms, Ann. Math. 121 (1985), 409-427. MR 86j:47061

7. R.Moore and T.Trent, Extreme points of certain operator algebras, Indiana U. Math. J. 36 (1987), 645-650. MR 89d:47103

8. B.Russo and H.Dye, A note on unitary operators in $C^{*}$-algebras, Duke Math. J. 33 (1966), 413-416. MR 33:1750

Department of Mathematics, Zhejiang University, Hangzhou 310027, People's RepubLIC OF CHINA

E-mail address: dongzhe@zju.edu.cn 